\title{
Expression of tumor necrosis factor- $\alpha$-induced protein 8 in stage III gastric cancer and the correlation with DcR3 and ERK1/2
}

\author{
RUYI HU ${ }^{1,2^{*}}$, WENMING LIU ${ }^{1,3^{*}}$, XINGFENG QIU $^{3}$, ZHENGHE LIN $^{3}$, YAN XIE $^{1}$, XINGYA HONG $^{4}$, \\ REYILA PAERHATI ${ }^{1}$, ZHONGQUAN QI $^{1}$, GUOHONG ZHUANG ${ }^{1}$ and ZHONGCHEN LIU ${ }^{1,5}$
}

\author{
${ }^{1}$ Organ Transplantation Institute, Anti-Cancer Research Center, Medical College, Xiamen University, Xiamen, Fujian 361100; \\ ${ }^{2}$ Department of General Surgery, The Ezhou Central Hospital, Ezhou, Hubei 436000; \\ ${ }^{3}$ Division of Gastroenterology Surgery, Zhongshan Hospital, Gastroenterology Institute of Xiamen University, \\ Gastroenterology Center of Xiamen, Xiamen, Fujian 361000; ${ }^{4}$ Department of Ultrasound, \\ Zhongshan Hospital of Xiamen University, Xiamen, Fujian 361100; ${ }^{5}$ Department of General Surgery, \\ The Tenth People's Hospital of Tongji University, Shanghai 200000, P.R. China
}

Received February 8, 2015; Accepted December 18, 2015

DOI: $10.3892 / \mathrm{ol} .2016 .4133$

\begin{abstract}
Tumor necrosis factor (TNF)- $\alpha$-induced protein 8 (TIPE) is a recently identified protein that is considered to be associated with various malignancies, including esophageal, breast and pancreatic cancer; however, the importance of TIPE in gastric cancer (GC) remains unknown. Decoy receptor 3 (DcR3) is a member of the tumor necrosis factor receptor superfamily that is expressed in digestive system neoplasms. The expression of DcR3 is regulated by the mitogen-activated protein kinase (MAPK)/MAPK kinase/extracellular signal-regulated kinase (ERK) signaling pathway. Reverse transcription-polymerase chain reaction was performed to detect the expression of TIPE, ERK and DcR3 in the pathological and tumor-adjacent normal gastric tissues of 30 patients that demonstrated stage III gastric adenocarcinoma. The expression and distribution of the TIPE protein was examined using immunohistochemistry, and the clinical significance and expression levels of DcR3 and ERK1/2 were evaluated. The expression of TIPE, ERK1/2 and DcR3 in the tumor tissues of GC was significantly increased compared with paracarcinoma tissues $(\mathrm{P}<0.05)$. In addition, TIPE expression positively correlated with DcR3 and ERK1 levels ( $r=0.538$ and
\end{abstract}

Correspondence to: Ms. Guohong Zhuang or Mr. Zhongchen Liu, Organ Transplantation Institute, Anti-Cancer Research Center, Medical College, Xiamen University, 168 Daxue Road, Xiamen, Fujian 361100, P.R. China

E-mail: zghxmu@163.com

E-mail: lzcxmu@163.com

${ }^{*}$ Contributed equally

Key words: GC, tumor necrosis factor- $\alpha$-induced protein 8 , decoy receptor 3, extracellular signal-regulated protein kinases 1 and 2 $\mathrm{r}=0.462$, respectively; $\mathrm{P}<0.05)$. There was no statistical difference between tumor tissues from patients with varying age, gender, differentiation or lymph node metastasis $(\mathrm{P}>0.05)$. TIPE may be vital in the progression of GC. TIPE may be associated with the expression of DcR3 and ERK1/2, which may be involved in the cell apoptosis of GC. The present study elucidates the potential function of TIPE as a novel marker and therapeutic target for GC.

\section{Introduction}

Gastric cancer (GC) is a leading cause of cancer-associated mortality worldwide, is responsible for 700,000 mortalities annually, and remains highly prevalent in numerous regions of Asia, Eastern Europe and South America (1). The majority of patients with GC are diagnosed at an advanced stage of disease, and the overall 5-year survival rate for patients with resectable GC remains low at $10-30 \%$, despite clinical advances in surgery and therapy $(2,3)$. The elucidation of molecules and signaling pathways that drive aggressive and metastatic GC is likely to offer novel avenues for the early identification and therapeutic intervention of GC.

Tumor necrosis factor (TNF)- $\alpha$-induced protein 8 (TIPE), also termed squamous cell carcinoma-stage 2 protein, cyclin G2-1 and nuclear factor- $\kappa \mathrm{B}$-inducible death effector domain-containing protein, was identified in a comparison between the expression profile of a primary human head and neck squamous cell carcinoma (HNSCC) cell line and the matching metastatic HNSCC-derived cell line (4). TIPE is a $21 \mathrm{kDa}$ cytosolic TNF- $\alpha$-inducible molecule and a member of the Fas-associated death domain-like interleukin-1 $\beta$-converting enzyme-like inhibitory protein family of cell death inhibitory proteins (5). The overexpression of TIPE contributes to enhanced DNA synthesis, cell proliferation and an inhibition of the activities of the apoptotic enzymes caspase 8 and caspase 3 (6,7). TIPE mRNA expression is induced by the activation of transcription factor nuclear factor $(\mathrm{NF})-\kappa \mathrm{B}$ 
and TNF- $\alpha$ in human cancer cells, vascular endothelial cells and primary rheumatoid arthritis synovial fibroblasts. The TNF- $\alpha$-mediated induction of TIPE mRNA may be reversed by $\mathrm{I} \kappa \mathrm{B} \alpha$, an inhibitor of $\mathrm{NF}-\kappa \mathrm{B}$, and the expression of TIPE in NF- $\mathrm{B}$-null cells inhibits TNF- $\alpha$-induced apoptosis (8). Collectively, the aforementioned functional studies indicate that TIPE is an oncogenic factor that is important for tumor progression, and that the exogenous expression of TIPE enhances cell survival.

With regard to the possible role of TIPE in tumorigenesis and tumor progression, TIPE overexpression has exhibited clinical relevance by frequently occurring in several types of cancer tissues, including in lung, colon, prostate and cervical cancer, esophageal squamous cell carcinoma and papillary thyroid carcinoma $(9,10)$. The exogenous expression of TIPE in tumor cells is associated with enhanced proliferation, cell migration and tumor growth (11). The knockdown of TIPE expression in tumor cells reduces tumorigenicity, which indicates that TIPE may be important in oncogenesis. External-signal regulated kinase (ERK) is a cytosolic receptor known to be pivotal in the nuclear protein import pathway in numerous cancers. The ERK pathway is linked to cellular proliferation, differentiation and apoptosis (12). Decoy receptor 3 (DcR3) is usually expressed in tumor cells and competitively inhibits TNF signaling. The overexpression of DcR3 in tumor cells protects the cells from apoptosis (13). Therefore, DcR3 and ERK are vital in the development of GC (14).

To the best of our knowledge, no studies on TIPE in GC have been previously reported. The binding partners and signaling pathways of TIPE are also unknown. In the present study, the expression pattern of TIPE in GC and paracarcinoma tissues was compared, the correlation between the expression levels of TIPE was analyzed, and the clinical significance and expression of ERK and DcR3 was assessed. The results indicated that TIPE may be important in the progression of GC.

\section{Patients and methods}

Patients and tissues. A total of $30 \mathrm{GC}$ tissues were obtained from 30 patients (27 male and 3 female) with stage III GC that had previously undergone complete resection at the Zhongshan Hospital (affiliated with Xiamen University; Xiamen, Fujian, China) between the years 2012 and 2013. The paracarcinoma tissue that was selected was located $\geq 5 \mathrm{~cm}$ away from the cancerous tissue. The tumor tissues from which the DNA was isolated were fresh specimens that were obtained during the resection surgery. DNA was isolated from the tissues $20 \mathrm{~min}$ after resection. None of the patients had received radiotherapy, chemotherapy or immunotherapy prior to the surgery. All samples were obtained with patient consent and approval of the Committee on Medical Ethics of Zhongshan Hospital, Xiamen University.

Follow-up information was obtained from a review of the patients' medical records. The mean age of the patients was 63.83 years (range, 43-88 years). The histological diagnosis and grade of the differentiation were evaluated using hematoxylin and eosin (H\&E; Beyotime Institute of Biotechnology, Shanghai, China)-stained sections, according to the World Health Organization Guidelines of Classification (15). All 30 specimens were revaluated with respect to histological
Table I. Primer sequences used for reverse transcription polymerase chain reaction.

\begin{tabular}{clc}
$\begin{array}{c}\text { Primer } \\
\text { pair }\end{array}$ & \multicolumn{1}{c}{ Sequence, 5'-3' } & $\begin{array}{c}\text { Fragment size, } \\
\text { base pairs }\end{array}$ \\
\hline TIPE & & 132 \\
F & CCCAGGGAAGTGGCTACAGA & \\
R & GCCTCCTTCTTGTTCTGGGT & 205 \\
ERK1 & & \\
F & CCTGCTCATCAACACCACC & \\
R & CGTAGCCACATACTCCGTCA & \\
ERK2 & & \\
F & TCTTCC AGCCCTCCTTCCTG & \\
R & CGTTTCTGCGCCGTTAGGT & \\
DcR3 & & \\
F & GCAAAGCCAAGGATTCCCCCTG & \\
R & GGCACTGCTCTGAGCTGGAGCTG & \\
$\beta$-actin & & \\
F & AGCCATGTACGTAGCCATCC & \\
R & ACCCTCATAGATGGGCACAG &
\end{tabular}

TIPE, tumor necrosis factor- $\alpha$-induced protein 8 ; F, forward; R, reverse; ERK1, extracellular signal-regulated protein kinase 1; ERK2, extracellular signal-regulated protein kinase 2; DcR3, decoy receptor 3 .

subtype, differentiation and tumor stage. The TNM staging system of Union for International Cancer Control was used to classify specimens (16).

Immunohistochemistry. The surgically excised tumor specimens were fixed with $10 \%$ neutral formalin (Shanghai Jianglai Bio-Technology Co., Ltd., Shanghai, China), embedded in paraffin (ZhongYou Chem, Xiamen, China), cut in a microtome (RM2235; Leica Biosystems Nussloch GmbH, Nussloch, Germany) to the desired thickness of $4 \mu \mathrm{m}$, and stained with $\mathrm{H} \& \mathrm{E}$ to confirm the tumor. Immunohistochemical staining for TIPE was performed using the streptavidin-peroxidase (ZSGB-BIO, Beijing, China) complex method. In brief, tissue sections were deparaffinized in xylene and rehydrated in a series of graded alcohol (100\%, $95 \%$ and $80 \%$ for $5 \mathrm{~min}$ ) using standard procedures. The sections were placed into an enamel cylinder (Shanghai Heqi Glassware Co., Ltd., Shanghai, China) that contained $10 \mathrm{mmol} / 1$ sodium citrate $(\mathrm{pH}$ 6.0; Sigma-Aldrich, St. Louis, MO, USA), which was heated using a gas burner at $95^{\circ} \mathrm{C}$ for 5 min for antigen unmasking. The sections were then immersed in $3 \%$ hydrogen peroxide (Sangon Biotech Co., Ltd., Shanghai, China) to remove the endogenous peroxidase. The sections were incubated with normal goat serum (Shanghai Haoran Bio Technologies Co., Ltd., Shanghai, China) to reduce nonspecific binding and then incubated at $4{ }^{\circ} \mathrm{C}$ overnight with rabbit anti-human TIPE polyclonal antibody (cat. no. ab166804; dilution, 1:200; Abcam, Cambridge, MA, USA). The sections were then washed with phosphate-buffered saline and incubated for 30 min with biotinylated goat anti-rabbit secondary antibody 
at $37^{\circ} \mathrm{C}$. The substrate $3,3^{\prime}$-diaminobenzidine tetrahydrochloride, which was dissolved in ultrapure water, was added in order to aid visualization of the expression.

RNA extraction and reverse transcription $(R T)$-polymerase chain reaction $(P C R)$. The total RNA was extracted from cells using TRIzol (Thermo Fisher Scientific, Waltham, MA, USA), in order to measure the TIPE/ERK/DcR3 gene copy number. The total RNA was extracted from the tissue using the TRIzol total RNA extraction reagent (CWbio, Beijing, China). The RNA quality and concentration were measured using a NanoDrop 2000 spectrophotometer (Thermo Fisher Scientific). RT and PCR were performed using the Takara PrimeScript ${ }^{\mathrm{TM}}$ RT-PCR kit (Takara Biotechnology Co., Ltd. Dalian, Liaoning, China) and the 2xEasyTaq ${ }^{\circledR}$ PCR super mix (TransGen, Beijing, China). Template cDNA was synthesized from $2.0 \mu \mathrm{g}$ of the total RNA using RT-PCR, which was performed using the Biometra TAdvanced Thermal Cycler (Biometra GmbH, Göttingen, Germany). Briefly, in a total volume of $20 \mu \mathrm{l}$, the samples were incubated at $94^{\circ} \mathrm{C}$ for $3 \mathrm{~min}$, followed by 30 cycles of incubation at $94^{\circ} \mathrm{C}$ for $30 \mathrm{sec}, 56^{\circ} \mathrm{C}$ for $30 \mathrm{sec}$ and $72^{\circ} \mathrm{C}$ for $1 \mathrm{~min}$, and then held at $72^{\circ} \mathrm{C}$ for $5 \mathrm{~min}$. The sequences of the primer pairs are shown in Table I. The RT-PCR results were analyzed using gel electrophoresis, the imaging outputs of which were compared using a grayscale analysis against the corresponding $\beta$-actin, and recorded for basic statistical analysis.

Statistical analysis. All analyses were performed using SPSS version 20.0 for Windows (IBM SPSS, Armonk, NY, USA). The data were presented as the mean \pm standard deviation. The significance of the difference between groups was assessed using the Student's two-tailed $t$-test. Spearman's rank correlation coefficient was used to analyze the correlation between TIPE, ERK and DcR3 expression. $\mathrm{P}<0.05$ was considered to indicate a statistically significant difference. All mean values were calculated from at least three independent experiments.

\section{Results}

Location of TIPE in stage III GC patients. To examine the distribution of TIPE, tumors from 30 patients were tested using immunohistochemistry. The results indicate that TIPE was expressed in the tumor tissues of the majority of the patients. TIPE expression was indicated in the cytoplasm and/or nucleolus of GC cells, and was not indicated in normal gastric mucosa (Fig. 1).

TIPE mRNA expression in stage III GC tissues. The analyses of the RT-PCR results were used to confirm the mRNA levels. The mean expression value of TIPE mRNA in cancer tissues $(0.7945 \pm 0.1451)$, which was normalized by $\beta$-actin gene expression, was significantly increased compared with the value in tumor-adjacent normal gastric tissues $(0.5421 \pm 0.0990 ; \mathrm{P}<0.05 ;$ comparative $t$-test $)$. There was a 1.5 -fold difference between the TIPE expression in the tumor and normal tissues (Fig. 2; Table II).

Association between TIPE MRNA expression and the clinicopathological characteristics of GC. In the present study,
Table II. Distribution of TIPE mRNA in the gastric tissues of 30 patients.

Type of tissue

TIPE mRNA value

Gastric cancer

$0.7945 \pm 0.1451$

Adjacent

$0.5421 \pm 0.0990$

$\mathrm{P}<0.05$ for the distribution of TIPE mRNA in two tissue types. TIPE, tumor necrosis factor- $\alpha$-induced protein 8 .

Table III. Distribution of TIPE mRNA in gastric cancer patients, according to clinicopathological characteristics.

\begin{tabular}{lrcr}
\hline Characteristics & $\begin{array}{c}\text { No. of } \\
\text { patients }\end{array}$ & $\begin{array}{c}\text { TIPE mRNA } \\
\text { value }\end{array}$ & P-value \\
\hline Gender & & & 0.532 \\
$\quad$ Male & 27 & $1.5218 \pm 0.6817$ & \\
Female & 3 & $2.2047 \pm 1.5754$ & \\
Age, years & & & 0.595 \\
$\quad<65$ & 17 & $1.6322 \pm 1.0004$ & \\
$65-75$ & 9 & $1.6806 \pm 0.4414$ & \\
$>75$ & 4 & $1.2069 \pm 0.1187$ & \\
Nodal status & & & 0.559 \\
N1 & 7 & $1.3865 \pm 0.4333$ & \\
N2 & 8 & $1.8311 \pm 1.4112$ & \\
N3 & 15 & $1.5565 \pm 0.4200$ & \\
Differentiation & & & 0.337 \\
Moderate & 11 & $1.4038 \pm 0.4502$ & \\
Poor & 19 & $1.6979 \pm 0.9333$ & \\
\hline
\end{tabular}

TIPE, tumor necrosis factor- $\alpha$-induced protein 8 .

Table IV. Distribution of DcR3, ERK1 and ERK2 mRNA in the gastric tissues of 30 patients.

\begin{tabular}{llcr}
\hline $\begin{array}{l}\text { Type of } \\
\text { mRNA }\end{array}$ & $\begin{array}{c}\text { Gastric } \\
\text { tissue }\end{array}$ & $\begin{array}{c}\text { mRNA } \\
\text { value }\end{array}$ & P-value \\
\hline DcR3 & Cancer & $2.1411 \pm 1.2424$ & 0.023 \\
& Normal & $1.8169 \pm 0.4850$ & \\
ERK1 & Cancer & $1.2497 \pm 0.6202$ & 0.033 \\
& Normal & $0.9656 \pm 0.6086$ & \\
ERK2 & Cancer & $1.1598 \pm 0.5080$ & 0.012 \\
& Normal & $0.8489 \pm 0.3659$ & \\
\hline
\end{tabular}

DcR3, decoy receptor 3; ERK1, extracellular signal-regulated protein kinase 1; ERK2, extracellular signal-regulated protein kinase 1.

all patients were diagnosed with pathological stage III GC; so the association of other pathological stages with TIPE was not analyzed. The distribution of TIPE status in GC according to the clinicopathological tests is reported in Table II. Table III 

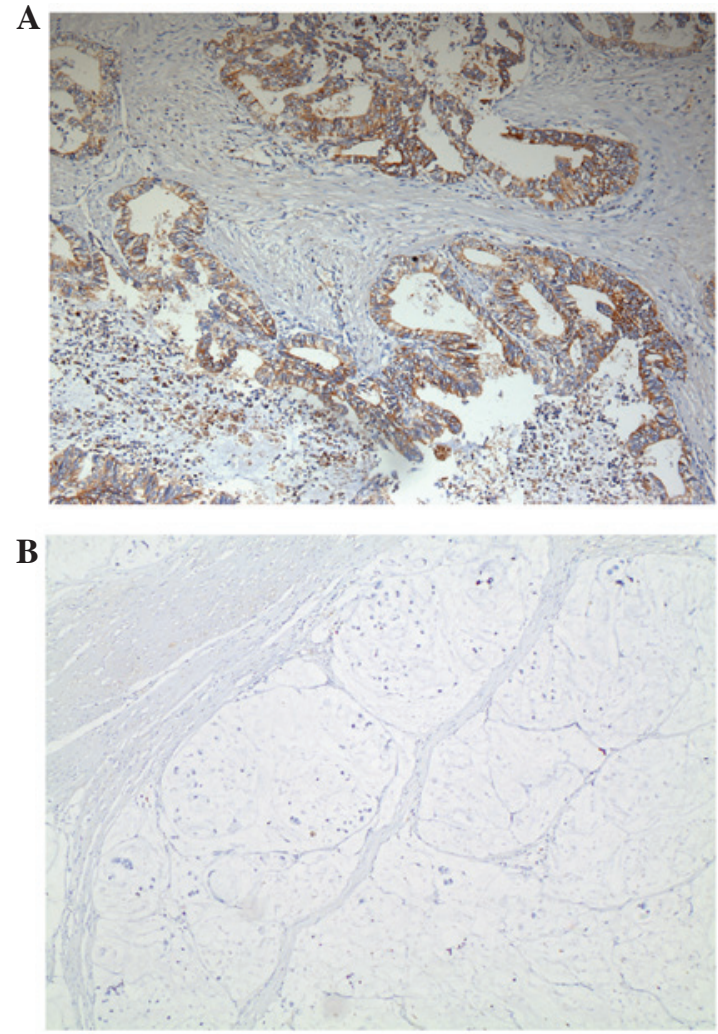

Figure 1. Location of tumor necrosis factor- $\alpha$-induced protein 8 in tissues analyzed using immunohistochemistry. (A) Stage III gastric cancer and (B) normal gastric mucosa (stain, 3,3'-diaminobenzidine tetrahydrochloride; magnification, x10).

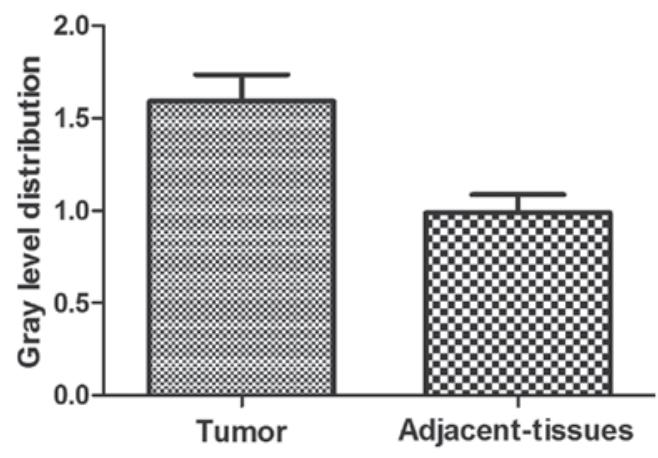

Figure 2. Expression of tumor necrosis factor- $\alpha$-induced protein 8 in stage III gastric cancer and normal tissues, analyzed using reverse transcription-polymerase chain reaction (normalized to $\beta$-actin).

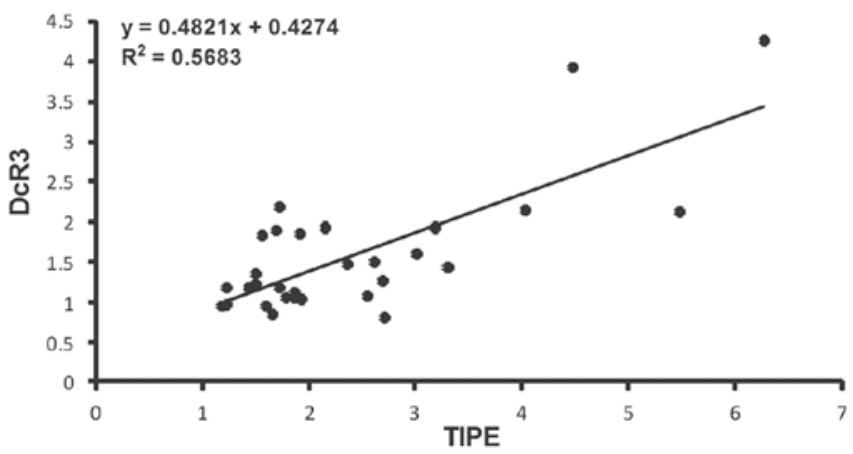

Figure 3. Correlation between TIPE and DcR3 expression. TIPE, tumor necrosis factor- $\alpha$-induced protein 8; DcR3, decoy receptor 3.

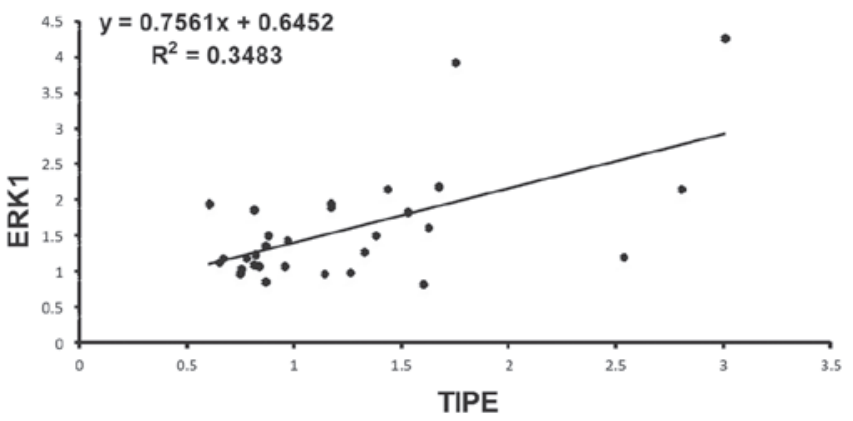

Figure 4. Correlation between TIPE and ERK1 expression. TIPE, tumor necrosis factor- $\alpha$-induced protein 8; ERK1, extracellular signal-regulated protein kinase 1.

shows that no strong association was observed between TIPE and gender, age, nodal status or differentiation $(\mathrm{P}>0.05)$.

The association of TIPE with DcR3 and ERK in GC. In order to identify the signaling molecules that are associated with TIPE, the expression of DcR3, ERK1 and ERK2 in GC tissues and tumor-adjacent normal gastric tissues was measured using RT-PCR. DcR3 mRNA and ERK1/2 mRNA was detected in tumor samples, and the mean expression value of DcR3 mRNA and ERK1/2 mRNA in cancer tissues was significantly increased compared with the value in non-cancerous tissues $(\mathrm{P}<0.05$; Table IV). Spearman's rank correlation coefficient analysis (Figs. 3 and 4) revealed that TIPE expression was positively correlated with DcR3 $(\mathrm{r}=0.733, \mathrm{P}<0.05)$ and ERK1 $(\mathrm{r}=0.590, \mathrm{P}<0.05)$ expression, but was not significantly correlated with ERK2 expression $(\mathrm{P}>0.05)$. In every case of high TIPE expression, DcR3 and ERK1 expression was increased compared with non-tumor tissues.

\section{Discussion}

To the best of our knowledge, the present study is the first to report that, unlike in normal gastric tissues, TIPE expression is upregulated at the protein and mRNA levels in GC tissues. In the present study, the cytoplasmic and nuclear localization and high expression levels of TIPE were not significantly associated with gender, age, nodal status or differentiation. In addition, TIPE overexpression was correlated with DcR3 and ERK1 expression in GC. The present study revealed a concomitant increase in TIPE and DcR3, ERK1 expression in cancer tissues, suggesting that TIPE may be important in the occurrence and progression of GC.

The increasing incidence, delayed detection and poor prognosis of advanced GC underline the requirement for novel diagnosis strategies, follow-up and treatment. The TIPE family is a novel subfamily of death effector domain proteins that consists of TIPE, TIPE1, TIPE2 and TIPE3, with TIPE being the first identified member $(17,18)$. TIPE was indicated to be expressed in various human cancer cell lines; in increased levels in A549 lung carcinoma, K562 chronic myelogenous leukemia and MOLT-4 lymphoblastic leukemia cells, and in lower levels in SW480 colorectal adenocarcinoma cells (19). TIPE overexpression was detected 
in several human malignant tumors at the mRNA and protein level. Positive correlations between TIPE and the histological grade, residual tumor size, recurrence and metastases of the peritoneum and lymph node have been demonstrated in the present study. In a previous study, TIPE overexpression was indicated to be an independent predictor of recurrent prostate cancer (8). In another study, functional variants of TIPE were hypothesized to result in tumor progression and poor outcomes for cervical cancer, by inhibiting the response of tumor cells to platinum drugs, particularly cisplatin and nedaplatin (13). Collectively, the aforementioned studies indicate that TIPE may be important in oncogenesis. However, the expression of TIPE and the correlation with clinical pathological factors has not been defined in GC.

In the present study, protein expression in TIPE was examined using immunohistochemistry, and mRNA levels were examined using RT-PCR in $30 \mathrm{GC}$ tissues. The results indicated that TIPE was expressed in the cytoplasm and nucleolus of GC cells, and that TIPE mRNA expression was significantly increased in GC compared with normal gastric tissues. The results were consistent with a previous finding that TIPE is overexpressed in human malignant tumors $(8-10,14)$. However, no association was observed between TIPE mRNA expression and nodal status or differentiation in the present study. Considering that the participants of the present study were all patients with stage III GC, additional studies of patients at various pathological stages of the disease are of paramount importance to confirming this conclusion, and if the sample size was increased, the findings may vary.

DcR3, also termed TR6, is a member of the tumor necrosis factor receptor superfamily, and is the decoy receptor for Fas ligand, tumor necrosis factor superfamily member 14 and TNF-like ligand 1A $(20,21)$. The DcR3 gene is expressed at a low levels in the human embryo, lung, brain, liver, spleen, stomach, colon, lymph nodes and spinal cord, and is expressed at a high levels in cancers, including gastrointestinal cancer, hepatocellular carcinoma and pancreatic cancer (22-24). DcR3 overexpression is associated with cell proliferation, lymph node metastasis, pathological stage and a significantly shortened survival rate $(13,25)$. Mitogen-activated protein kinases (MAPKs) are an important group of serine and threonine signaling kinases that activate certain transcription factors by causing a cascade of protein phosphorylation through a variety of extracellular stimuli. In addition, the extracellular signal-regulated kinase (ERK) pathway is linked to cellular proliferation and differentiation and the proinflammatory cellular response. Previously, evidence has indicated that the expression of ERK1/2 was increased in certain cancers, which correlated with the TNM stages and prognosis $(26,27)$. In the present study, the expression of DcR3 and ERK1/2 mRNA was detected in the same samples using RT-PCR. The results indicated that the level of DcR3 and ERK1/2 mRNA in GC tissues is significantly increased compared with normal gastric tissues. Additionally, TIPE expression positively correlated with DcR3 and ERK1 when analyzed with Spearman's rank correlation coefficient. Therefore, the samples that demonstrated high TIPE mRNA expression also demonstrated high DcR3 and ERK1 expression in stage III GC tissues.

The present findings indicate that TIPE may be important in the oncogenesis and progression of GC. TIPE may, therefore, be considered as a novel marker and promising therapeutic target in GC patients. However, the present study has a number of limitations. The present study is a retrospective analysis and studies a limited number of patients. The protein expression was not examined using western blot analysis, and the mechanism behind the findings was not examined due to limited time. Therefore additional studies are required in order to examine a larger sample of patients, and to explore the possible mechanism of GC oncogenesis and progression caused by the overexpression of TIPE.

\section{Acknowledgements}

This study was supported by the grants from the National Natural Science Foundation of China (grant no. 81272720), Public Projects of Fujian Province (grant no. 2016R1102) Fujian Province Health Planning Commission medical innovation subject (grant no. 2014-CXB-43).

\section{References}

1. Brenner H, Rothenbacher D and Arndt V: Epidemiology of stomach cancer. Methods Mol Biol 472: 467-477, 2009.

2. Hartgrink HH, Jansen EP, van Grieken NC and van de Velde CJ: Gastric cancer. Lancet 374: 477-490, 2009.

3. Bang YJ, Van Cutsem E, Feyereislova A, Chung HC, Shen L, Sawaki A, Lordick F, Ohtsu A, Omuro Y, Satoh T, et al; ToGA Trial Investigators: Trastuzumab in combination with chemotherapy versus chemotherapy alone for treatment of HER2-positive advanced gastric or gastro-oesophageal junction cancer (ToGA): A phase 3, open-label, randomised controlled trial. Lancet 376: 687-697, 2010.

4. Patel S, Wang FH, Whiteside TL and Kasid U: Identification of seven differentially displayed transcripts in human primary and matched metastatic head and neck squamous cell carcinoma cell lines: Implications in metastasis and/or radiation response. Oral Oncol 33: 197-203, 1997.

5. Kumar D, Gokhale P, Broustas C, Chakravarty D, Ahmad I and Kasid U: Expression of SCC-S2, an antiapoptotic molecule, correlates with enhanced proliferation and tumorigenicity of MDA-MB 435 cells. Oncogene 23: 612-616, 2004.

6. You Z, Ouyang H, Lopatin D, Polver PJ and Wang CY: Nuclear factor-kappa B-inducible death effector domain-containing protein suppresses tumor necrosis factor-mediated apoptosis by inhibiting caspase-8 activity. J Biol Chem 276: 26398-26404, 2001.

7. Laliberté B, Wilson AM, Nafisi H, Mao H, Zhou YY, Daigle M and Albert PR: TNFAIP8: A new effector for Galpha(i) coupling to reduce cell death and induce cell transformation. J Cell Physiol 225: 865-874, 2010.

8. Zhang C, Kallakury BV, Ross JS, Mewani RR, Sheehan CE, Sakabe I, Luta G, Kumar D, Yadavalli S, Starr J, et al: The significance of TNFAIP8 in prostate cancer response to radiation and docetaxel and disease recurrence. Int J Cancer 133: 31-42, 2013.

9. Dong QZ, Zhao Y, Liu Y, Wang Y,Zhang PX, Jiang GY, Dong XJ, Cui QZ and Wang EH: Overexpression of SCC-S2 correlates with lymph node metastasis and poor prognosis in patients with non-small-cell lung cancer. Cancer Sci 101: 1562-1569, 2010.

10. Duan D, Zhu YQ, Guan LL and Wang J: Upregulation of SCC-S2 in immune cells and tumor tissues of papillary thyroid carcinoma. Tumour Biol 35: 4331-4337, 2014.

11. Laliberté B, Wilson AM, Nafisi H, Mao H, Zhou YY, Daigle M and Albert PR: TNFAIP8: A new effector for Galpha(i) coupling to reduce cell death and induce cell transformation. J Cell Physiol 225: 865-874, 2010.

12. Huang D, Ding Y, Luo WM, Bender S, Qian CN, Kort E, Zhang ZF, VandenBeldt K, Duesbery NS, Resau JH and Teh BT: Inhibition of MAPK kinase signaling pathways suppressed renal cell carcinoma growth and angiogenesis in vivo. Cancer Res 68: 81-88, 2008.

13. Wu Y, Guo E, Yu J, Xie Q and Chen J: High DcR3 expression predicts stage $\mathrm{pN} 2$ in GC. Hepatogastroenterology 54: 2172-2176, 2007. 
14. Yang D, Fan X, Yin P, Wen Q, Yan F, Yuan S, Liu B, Zhuang G and Liu Z: Significance of decoy receptor 3 (Dcr3) and external-signal regulated kinase $1 / 2($ Erk1/2) in gastric cancer. BMC Immunol 13: 28, 2012.

15. Kleihues P and Sobin LH: World Health Organization classification of tumours. Cancer 88: 2887, 2000.

16. Sobin LH and Wittekind C (eds): International Union Against Cancer (UICC). TNM Classification of Malignant Tumours. 6th edition. Wiley Blackwell, Hoboken, New Jersey, 2002.

17. Freundt EC, Bidere $\mathrm{N}$ and Lenardo MJ: A different TIPE of immune homeostasis. Cell 133: 401-402, 2008.

18. Sun H, Gong S, Carmody RJ, Hilliard A, Li L, Sun J, Kong L, Xu L, Hilliard B, Hu S, Shen H, Yang X and Chen YH: TIPE2, a negative regulator of innate and adaptive immunity that maintains immune homeostasis. Cell 133: 415-426, 2008.

19. Kumar D, Whiteside TL and Kasid U: Identification of a novel tumor necrosis factor-alpha-inducible gene, SCC-S2, containing the consensus sequence of a death effector domain of fas-associated death domain-like interleukin- 1beta-converting enzyme-inhibitory protein. J Biol Chem 275: 2973-2978, 2000.

20. Migone TS, Zhang J, Luo X, Zhuang L, Chen C, Hu B, Hong JS Perry JW, Chen SF, Zhou JX, et al: TL1A is a TNF-like ligand for DR3 and TR6/DcR3 and functions as a T cell costimulator. Immunity 16: 479-492, 2002.

21. Roth W, Isenmann S, Nakamura M, Platten M, Wick W, Kleihues P, Bähr M, Ohgaki H, Ashkenazi A and Weller M: Soluble decoy receptor 3 is expressed by malignant gliomas and suppresses CD95 ligand-induced apoptosis and chemotaxis. Cancer Res 61: 2759-2765, 2001.
22. Pitti RM, Marsters SA, Lawrence DA, Roy M, Kischkel FC, Dowd P, Huang A, Donahue CJ, Sherwood SW, Baldwin DT, et al: Genomic amplification of a decoy receptor for Fas ligand in lung and colon cancer. Nature 396: 699-703, 1998.

23. Bai C, Connolly B, Metzker ML, Hilliard CA, Liu X, Sandig V, Soderman A, Galloway SM, Liu Q, Austin CP and Caskey CT: Overexpression of M68/DcR3 in human gastrointestinal tract tumors independent of gene amplification and its location in a four-gene cluster. Proc Natl Acad Sci USA 97: 1230-1235, 2000.

24. Tsuji S, Hosotani R, Yonehara S, Masui T, Tulachan SS, Nakajima S, Kobayashi H, Koizumi M, Toyoda E and Ito D: Endogenous decoy receptor 3 blocks the growth inhibition signals mediated by Fas ligand in human pancreatic adenocarcinoma. Int J Cancer 106: 17-25, 2003.

25. Yu KY, Kwon B, Ni J, Zhai Y, Ebner R and Kwon BS: A newly identified member of tumor necrosis factor receptor superfamily (TR6) suppresses LIGHT-mediated apoptosis. J Biol Chem 274: 13733-13736, 1999.

26. Song H, Lee AY, Jung H, Choi JH, Roh K, Ha S, Kim KD, Bae KB, Kang MS, Park S, et al: A8, an anti-uPA agonistic antibody, promotes metastasis of cancer cells via ERK pathway. Monoclon Antib Immunodiagn Immunother 33: 312-318, 2014.

27. Jerjees DA1, Alabdullah M, Alkaabi M, Abduljabbar R, Muftah A, Nolan C, Green AR, Ellis IO and Rakha EA: ERK1/2 is related to oestrogen receptor and predicts outcome in hormone-treated breast cancer. Breast Cancer Res Treat 147: 25-37, 2014. 